\title{
WILD PRIMES OF A HIGHER DEGREE SELF-EQUIVALENCE OF A NUMBER FIELD
}

\author{
Alfred Czogata, Beata Rothkegel, Andrzej SŁadek
}

\begin{abstract}
Let $\ell>2$ be a prime number. Let $K$ be a number field containing a unique $\ell$-adic prime and assume that its class is an $\ell$ th power in the class group $C_{K}$. The main theorem of the paper gives a sufficient condition for a finite set of primes of $K$ to be the wild set of some Hilbert self-equivalence of $K$ of degree $\ell$.
\end{abstract}

\section{Introduction}

Let $n>1$ be a natural number and let $K$ as well as $L$ be number fields containing a primitive $n$th root of unity. A Hilbert symbol equivalence of degree $n$ between $K$ and $L$ is defined as a triple

$$
f: \mu_{n}(K) \rightarrow \mu_{n}(L), \quad T: \Omega(K) \rightarrow \Omega(L), \quad t: \dot{K} / \dot{K}^{n} \rightarrow \dot{L} / \dot{L}^{n},
$$

where $f$ is an isomorphism between the groups of $n$th roots of unity, $t$ is an isomorphism between the $n$th power class groups and $T$ is a bijection between the sets of all primes of $K$ and $L$ with $(f, T, t)$ preserving Hilbert symbols of degree $n$ in the sense that

$$
(a, b)_{\mathfrak{p}}^{f}=(t a, t b)_{T \mathfrak{p}} \quad \text { for all } \quad a, b \in \dot{K} / \dot{K}^{n}, \mathfrak{p} \in \Omega(K) .
$$

Received: 5.04.2016. Revised: 22.05.2016. Accepted: 01.06.2016.

(2010) Mathematics Subject Classification: 11E12, $11 \mathrm{E} 81$.

Key words and phrases: higher degree Hilbert-symbol equivalence, wild prime. 
In the case $K=L$ and $f=\mathrm{id}$, the Hilbert equivalence $(f, T, t)$ is called Hilbert self-equivalence of degree $n$ of $K$ or just self-equivalence of degree $n$ of $K$ and denoted by $(T, t)$.

Hilbert equivalence of degree 2 was introduced in [11] as a necessary and sufficient condition for Witt equivalence of two global fields. The main result of [11] states that two global fields of characteristic not 2 have isomorphic Witt rings if and only if there exists a Hilbert equivalence of degree 2 between these fields.

Hilbert equivalence of degree $n>2$, was introduced in [2] and was used for a classification of Milnor rings modulo $n$. In [16] it was proven that for two number fields containing nth roots of unity, $n$ square free, there exists a Hilbert equivalence of degree $n$ if and only if there exists an isomorphism of graded rings $\mathrm{K}(K) / n \mathrm{~K}(K) \cong \mathrm{K}(L) / n \mathrm{~K}(L)$ such that $\{-1\}_{n} \longmapsto\{-1\}_{n}$, where $\mathrm{K}(F)$ denotes the Milnor ring of $F$, (cf. [9]).

A finite prime $\mathfrak{p} \in \Omega(K)$ is called a tame prime of the Hilbert equivalence $(f, T, t)$ of degree $n$ if

$$
\operatorname{ord}_{\mathfrak{p}} a \equiv \operatorname{ord}_{T \mathfrak{p}} t a \quad(\bmod n) \quad \text { for all } \quad a \in \dot{K} / \dot{K}^{n} .
$$

A finite prime $\mathfrak{p} \in \Omega(K)$ is said to be wild if it is not a tame prime of $(f, T, t)$. The set $\mathcal{W}(f, T, t)$ of all wild primes of $(f, T, t)$ is called the wild set of $(f, T, t)$. The Hilbert equivalence $(f, T, t)$ is said to be tame if all finite primes are tame. In [5] and [4] the reader can find characterizations of tame Hilbert equivalence of degree 2 and degree $\ell>2, \ell$ being a prime number, respectively.

In the case of a Hilbert equivalence which is not tame it is natural to ask what the wild set of this equivalence could be. In [13] Somodi estimated the size of the wild set of Hilbert equivalence of degree 2, whereas in [14] and [15] he gave a description of the wild set of Hilbert self-equivalence of degree 2 of the rational number field $\mathbb{Q}$ as well as the Gaussian field $\mathbb{Q}(i)$. Somodi's results were generalized to a wider family of number fields in [7].

In this paper we consider self-equivalences of degree $\ell$, where $\ell$ is a prime number $\neq 2$, of number fields $K$ satisfying two conditions:

(C1) The field $K$ contains a primitive $\ell$ th root of unity.

(C2) The field $K$ has exactly one $\ell$-adic prime $\mathfrak{r}$ and its class $\mathrm{cl} \mathfrak{r}$ is an $\ell$ th power in the ideal class group $C_{K}$ of $K$.

For a number field $K$ satisfying $(\mathrm{C} 1)$ and $(\mathrm{C} 2)$ we find a sufficient condition for a finite set of finite primes of $K$ to be the wild set of some Hilbert selfequivalence of degree $\ell$ of $K$.

The main result of the paper is as follows.

THEOREM 1.1. Let $\ell>2$ be a prime number and let $K$ be a number field satisfying $(\mathrm{C} 1)$ and $(\mathrm{C} 2)$. If $W=\left\{\mathfrak{p}_{1}, \ldots, \mathfrak{p}_{k}\right\}$ is a set of finite primes of $K$ 
and the classes $\operatorname{cl} \mathfrak{p}_{1}, \ldots \mathrm{cl} \mathfrak{p}_{k}$ are $\ell$ th powers in $C_{K}$, then there exists a Hilbert self-equivalence $(T, t)$ of degree $\ell$ of $K$ such that $\mathcal{W}(T, t)=W$.

In the proof we use the technique developed in [3] and [4]. Basic facts and notions connected with this technique are presented in Sections 2 and 3.

The main theorem is proven in two steps. In the first one, presented in Section 3, we construct a Hilbert self-equivalence of degree $\ell$ with one wild prime adjusting the construction applied in [7] to the case of $\ell>2$. The second step, carried out in Section 4, uses the analysis of the behaviour of wild sets under a composition of self-equivalences of degree $\ell$.

\section{Singular elements}

Throughout the paper, let $\ell>2$ be a prime number, $K$ be a number field and $\zeta \in K$ be a fixed primitive $\ell$ th root of unity. For any $\mathfrak{p} \in \Omega(K)$ we write $K_{\mathfrak{p}}$ for the completion of $K$ at $\mathfrak{p},(\cdot, \cdot)_{\mathfrak{p}}: \dot{K}_{\mathfrak{p}} \times \dot{K}_{\mathfrak{p}} \rightarrow \mu_{\ell}(K)$ for the Hilbert symbol of degree $\ell$ with values in the cyclic group $\mu_{\ell}(K)$ generated by $\zeta$ and $(\dot{\bar{p}})_{\ell}$ for the $\ell$ th power residue symbol. Since $\left(x_{1}, x_{2}\right)_{\mathfrak{p}}=\left(y_{1}, y_{2}\right)_{\mathfrak{p}}$, if $x_{i} \dot{K}_{\mathfrak{p}}^{\ell}=y_{i} \dot{K}_{\mathfrak{p}}^{\ell}, i=1,2$, we may consider $(\cdot, \cdot)_{\mathfrak{p}}$ as a mapping defined on $\dot{K}_{\mathfrak{p}} / \dot{K}_{\mathfrak{p}}^{\ell} \times \dot{K}_{\mathfrak{p}} / \dot{K}_{\mathfrak{p}}^{\ell}$

If $A$ is a finite abelian group, then the quotient $A / A^{\ell}$ is an elementary abelian $\ell$-group. The group $A / A^{\ell}$ can be equipped with the structure of a linear space over the $\ell$-element field $\mathbb{F}_{\ell}$. In this sense we will use the notion of linear independence of elements of $A / A^{\ell}$. The $\ell$-rank $\mathrm{rk}_{\ell} A$ of a group $A$ is $\operatorname{dim}_{\mathbb{F}_{\ell}} A / A^{\ell}$. Where it is not misleading, we will use frequently the same symbol for $x \in A$ and for its canonical image in $A / A^{\ell}$. We write $\left\langle x_{1}, \ldots x_{n}\right\rangle$ for the subgroup of $A$ generated by the elements $x_{1}, \ldots, x_{n} \in A$.

A finite nonempty set $S \subset \Omega(K)$ is called a Hasse set if it contains all infinite primes of $K$. We write $\mathcal{O}_{S}, U_{S}, C_{S}, h^{S}$ for the ring of $S$-integers, the group of $S$-units, the $S$-class group of $K$ and the order of $C_{S}$, respectively. The class of the ideal $\mathfrak{a}$ of $\mathcal{O}_{S}$ in the group $C_{S}$ we denote by cl a.

A Hasse set $S \subset \Omega(K)$ is said to be sufficiently large, if:

- $S$ contains all $\ell$-adic primes of $K$,

- the $S$-class number $h^{S}$ is not divisible by $\ell$, i.e. $\operatorname{rk}_{\ell} C_{S}=0$.

For a Hasse set $S$ of $K$ we denote

$$
E_{S}=\left\{a \in \dot{K}: \operatorname{ord}_{\mathfrak{p}} a \equiv 0(\bmod \ell), \quad \forall \mathfrak{p} \in \Omega(K) \backslash S\right\} .
$$


It is easy to check that $E_{S}$ is a subgroup of the multiplicative group $\dot{K}$. Elements of $E_{S}$ are called $S$-singular.

We identify elements of the group $U_{S} / U_{S}^{\ell}$ with their images under the natural embedding $U_{S} / U_{S}^{\ell} \rightarrow E_{S} / \dot{K}^{\ell}$, so we may consider $U_{S} / U_{S}^{\ell}$ as a subgroup of the group $E_{S} / \dot{K}^{\ell}$. By Dirichlet Unit Theorem its $\ell$-rank equals

$$
\operatorname{rk}_{\ell} U_{S} / U_{S}^{\ell}=\# S
$$

Proposition 2.1. If $S$ is a Hasse set of primes of $K$, then

$$
\mathrm{rk}_{\ell} E_{S} / \dot{K}^{\ell}=\# S+\mathrm{rk}_{\ell} C_{S}(K) .
$$

Proof. The mapping

$$
E_{S} \rightarrow{ }_{\ell} C_{S}, \quad x \mapsto \mathrm{cl} \prod_{\mathfrak{p} \notin S} \mathfrak{p}^{\frac{1}{\ell} \operatorname{ord}_{\mathfrak{p}} x}
$$

is a group epimorphism with the kernel $U_{S} \dot{K}^{\ell}$. It suffices to notice that $U_{S} \dot{K}^{\ell} / \dot{K}^{\ell} \cong U_{S} / U_{S}^{\ell}$ and then apply 2.1.

Contraction of the diagonal homomorphism $\operatorname{diag}_{S}: \dot{K} / \dot{K}^{\ell} \rightarrow \prod_{\mathfrak{p} \in S} \dot{K}_{\mathfrak{p}} / \dot{K}_{\mathfrak{p}}^{\ell}$ to the subgroup $E_{S} / \dot{K}^{\ell}$, gives the homomorphism $E_{S} / \dot{K}^{\ell} \rightarrow \prod_{\mathfrak{p} \in S} \dot{K}_{\mathfrak{p}} / \dot{K}_{\mathfrak{p}}^{\ell}$, which we denote by $i_{S}$.

Let

$$
\Delta_{S}=\left\{a \in E_{S}: a \in \dot{K}_{\mathfrak{p}}^{\ell}, \quad \forall \mathfrak{p} \in S\right\} .
$$

The group $\Delta_{S} / \dot{K}^{\ell}$ is the kernel of $i_{S}$.

Remark 2.2. Suppose $S \subset S^{\prime}$ are Hasse sets of $K$. Then $E_{S} \subseteq E_{S^{\prime}}$, $\Delta_{S^{\prime}} \subseteq \Delta_{S}$ and there exists a natural group epimorphism $C_{S} \rightarrow C_{S^{\prime}}$, which induces the group epimorphism $C_{S} / C_{S}^{\ell} \rightarrow C_{S^{\prime}} / C_{S^{\prime}}^{\ell}$ with the kernel equal to the subgroup of $C_{S} / C_{S}^{\ell}$ generated by the set $\left\{\operatorname{cl} \mathfrak{p} C_{S}^{\ell}: \mathfrak{p} \in S^{\prime} \backslash S\right\}$. Thus

$$
\operatorname{rk}_{\ell} C_{S^{\prime}}=\operatorname{rk}_{\ell} C_{S}-\operatorname{rk}_{\ell}\left\langle\left\{\operatorname{clp} C_{S}^{\ell}: \mathfrak{p} \in S^{\prime} \backslash S\right\}\right\rangle
$$

and

$$
\mathrm{rk}_{\ell} E_{S^{\prime}} / \dot{K}^{\ell}=\# S^{\prime}+\left(\mathrm{rk}_{\ell} C_{S}-\operatorname{rk}_{\ell}\left\langle\left\{\operatorname{clp} C_{S}^{\ell}: \mathfrak{p} \in S^{\prime} \backslash S\right\}\right\rangle\right) .
$$


LEMma 2.3. Let $S$ be a Hasse set of $K$ containing all $\ell$-adic primes and let $\mathfrak{p} \in \Omega(K) \backslash S$. Then

$$
\operatorname{cl} \mathfrak{p} \in C_{S}^{\ell} \Longleftrightarrow\left(\frac{b}{\mathfrak{p}}\right)_{\ell}=1 \quad \text { for every } b \in \Delta_{S} .
$$

Proof. $(\Rightarrow)$ By assumption there exists $x_{\mathfrak{p}} \in \dot{K}$ such that $x_{\mathfrak{p}} \mathcal{O}_{S}=\mathfrak{p} \cdot J^{\ell}$ for some $S$-ideal $J$ of the field $K$. Fix $b \in \Delta_{S}$. Since for every prime $\mathfrak{q} \notin S \cup\{\mathfrak{p}\}$ the elements $b$ and $x_{\mathfrak{p}}$ are $\mathfrak{q}$-adic units modulo $\dot{K}_{\mathfrak{q}}^{\ell}$, we have

$$
\left(b, x_{\mathfrak{p}}\right)_{\mathfrak{q}}=1 \quad \text { for all } \mathfrak{q} \notin S \cup\{\mathfrak{p}\} .
$$

Also

$$
\left(b, x_{\mathfrak{p}}\right)_{\mathfrak{q}}=1 \text { for all } \mathfrak{q} \in S,
$$

because $b \in \dot{K}_{\mathfrak{q}}^{\ell}$ for all $\mathfrak{q} \in S$.

By Hilbert reciprocity law, $\left(b, x_{\mathfrak{p}}\right)_{\mathfrak{p}}=1$, i.e. $\left(\frac{b}{\mathfrak{p}}\right)_{\ell}=1$.

$(\Leftarrow)$ Let $S_{1}=S \cup\{\mathfrak{p}\}$. Since $b \in \dot{K}_{\mathfrak{p}}^{\ell}$ for every $b \in \Delta_{S}$ (by assumption), $\Delta_{S_{1}}=\Delta_{S}$. Thus $\operatorname{rk}_{\ell} C_{S}=\operatorname{rk}_{\ell} C_{S_{1}}$, and $\operatorname{clp} \in C_{S}^{\ell}$.

Lemma 2.4. Let $S$ be a Hasse set of $K$ containing all $\ell$-adic primes and let $\mathfrak{q}_{1}, \ldots, \mathfrak{q}_{m} \in \Omega(K) \backslash S$ be finite primes. Then the classes $\mathrm{cl} \mathfrak{q}_{1}, \ldots, \mathrm{cl} \mathfrak{q}_{m}$ are linearly independent in the factor group $C_{S} / C_{S}^{\ell}$ if and only if there exist elements $b_{1}, \ldots, b_{m} \in \Delta_{S}$ linearly independent in the group $\Delta_{S} / \dot{K}^{\ell}$ such that

$$
\left(\frac{b_{i}}{\mathfrak{q}_{i}}\right)_{\ell}=\zeta, \quad\left(\frac{b_{j}}{\mathfrak{q}_{i}}\right)_{\ell}=1 \quad \text { for all } i, j \in\{1, \ldots, m\}, i \neq j .
$$

Proof. $(\Leftarrow)$ Suppose the classes $\mathfrak{q}_{1}, \ldots, \mathfrak{q}_{m}$ are linearly dependent in the group $C_{S} / C_{S}^{\ell}$. Then

$$
x \mathcal{O}_{S}(K)=\mathfrak{q}_{1}^{\epsilon_{1}} \ldots \mathfrak{q}_{m}^{\epsilon_{m}} \mathfrak{I}^{\ell}
$$

for some element $x \in \dot{K}$, fractional $S$-ideal $\mathfrak{I}$ and $\epsilon_{1}, \ldots, \epsilon_{m} \in\{0, \ldots, \ell-1\}$ not all equal to zero.

We may assume $\epsilon_{1}>0$. The element $b_{1}$ is a $\mathfrak{q}_{j}$-adic unit (modulo $\dot{K}_{\mathfrak{q}_{j}}^{\ell}$ ) for every $j \in\{1, \ldots, m\}$, so by the well known relationship between Hilbert 
symbol of degree $\ell$ and $\ell$ th power residue symbol (cf. [10]) we get the following equalities

$$
\left(b_{1}, x\right)_{\mathfrak{q}_{1}}=\left(\frac{b_{1}}{\mathfrak{q}_{1}}\right)_{\ell}^{\operatorname{ord}_{\mathfrak{q}_{1}}(x)}=\zeta^{\epsilon_{1}} \neq 1, \quad\left(b_{1}, x\right)_{\mathfrak{q}_{j}}=\left(\frac{b_{1}}{\mathfrak{q}_{j}}\right)_{\ell}^{\operatorname{ord}_{\mathfrak{q}_{j}}(x)}=1
$$

for every $j \in\{2, \ldots, m\}$.

Since $b_{1} \in \dot{K}_{\mathfrak{p}}^{\ell}$ for every $\mathfrak{p} \in S$, we have $\left(b_{1}, x\right)_{\mathfrak{p}}=1$. Every prime $\mathfrak{q}$ of $K$ not in $S \cup\left\{\mathfrak{q}_{1}, \ldots, \mathfrak{q}_{m}\right\}$ is a finite non- $\ell$-adic prime and the elements $x, b_{1}$ are $\mathfrak{q}$-adic units (modulo $\dot{K}_{\mathfrak{q}}^{\ell}$ ), so $\left(b_{1}, x\right)_{\mathfrak{q}}=1$. This contradicts Hilbert reciprocity law.

$(\Rightarrow)$ We use induction on $m$. For $m=1$ the statement follows from Lemma 2.3.

Suppose $m>1$. By Lemma 2.3 there exists $b_{1} \in \Delta_{S}$ such that

$$
\left(\frac{b_{1}}{\mathfrak{q}_{1}}\right)_{\ell}=\zeta
$$

Let $S_{1}=S \cup\left\{\mathfrak{q}_{1}\right\}$. Then $\operatorname{rk}_{\ell} C_{S_{1}}=\operatorname{rk}_{\ell} C_{S}-1, \quad \Delta_{S_{1}} \subseteq \Delta_{S}$ and $b_{1} \notin \Delta_{S_{1}}$. Moreover, the classes $\mathrm{cl} \mathfrak{q}_{2}, \ldots, \operatorname{cl} \mathfrak{q}_{m}$ are linearly independent in the group $C_{S_{1}} / C_{S_{1}}^{\ell}$.

By induction hypothesis we may find $b_{2}, \ldots, b_{m} \in \Delta_{S_{1}}$, linearly independent in the group $\Delta_{S_{1}} / \dot{K}^{\ell}$, such that

$$
\left(\frac{b_{i}}{\mathfrak{q}_{i}}\right)_{\ell}=\zeta, \quad\left(\frac{b_{i}}{\mathfrak{q}_{j}}\right)_{\ell}=1 \quad \text { for all } i, j \in\{2, \ldots, m\}, i \neq j .
$$

Obviously, $\left(\frac{b_{i}}{\mathfrak{q}_{1}}\right)_{\ell}=1$ for $i=2, \ldots, m$. If necessary, we multiply $b_{1}$ by a product of appropriate powers of $b_{i}, i \in\{2, \ldots, m\}$, to get $\left(\frac{b_{1}}{\mathfrak{q}_{i}}\right)_{\ell}=1$ for $i=2, \ldots, m$.

Corollary 2.5. Let $S$ be a Hasse set of primes of $K$ containing all $\ell$-adic primes and let $\mathfrak{q}_{1}, \ldots, \mathfrak{q}_{m} \in \Omega(K) \backslash S$ be finite primes such that $\mathrm{cl} \mathfrak{q}_{1}, \ldots, \mathrm{cl} \mathfrak{q}_{m}$ is a basis of the factor group $C_{S} / C_{S}^{\ell}$. Then

$$
E_{S} / \dot{K}^{\ell}=U_{S} \dot{K}^{\ell} / \dot{K}^{\ell} \oplus \Delta_{S} / \dot{K}^{\ell}=U_{S} \dot{K}^{\ell} / \dot{K}^{\ell} \oplus\left\langle b_{1} \dot{K}^{\ell}, \ldots, b_{m} \dot{K}^{\ell}\right\rangle,
$$

where $b_{1}, \ldots, b_{n}$ are chosen as in the lemma above. 


\section{Hilbert symbol bilinear spaces}

In this section we will recall basic properties of Hilbert symbol of degree $\ell$ and prove new facts which will appear to be crucial in the next sections. Let us keep the assumptions on $\ell, K$ and $\zeta$ made at the beginning of the previous section.

Since $\ell>2$, every infinite prime $\mathfrak{p}$ of $K$ is complex and the $\mathfrak{p}$-adic Hilbert symbol is trivial, i.e. $(a, b)_{\mathfrak{p}}=1$ for all $a, b \in \dot{K}_{\mathfrak{p}}$.

If $\mathfrak{p}$ is a finite and non- $\ell$-adic prime, then according to the formula for the value of Hilbert symbol (cf. [10, Theorem 5.4]) we have:

3.1. If $u, v$ are $\mathfrak{p}$-adic units, then $(u, v)_{\mathfrak{p}}=1$.

3.2. If $u$ is a $\mathfrak{p}$-adic unit and $\pi_{\mathfrak{p}}$ is a uniformizer, i.e. $\operatorname{ord}_{\mathfrak{p}} \pi_{\mathfrak{p}}=1$, then

$$
\left(\pi_{\mathfrak{p}}, u\right)_{\mathfrak{p}}=1 \Longleftrightarrow u \in U_{\mathfrak{p}}^{\ell}
$$

3.3. There exists a $\mathfrak{p}$-adic unit $u_{\mathfrak{p}}$ such that

$$
\left(u_{\mathfrak{p}}, x\right)_{\mathfrak{p}}=\zeta^{\operatorname{ord}_{\mathfrak{p}} x} \quad \text { for every } x \in \dot{K}_{\mathfrak{p}}
$$

For an $\ell$-adic prime $\mathfrak{p}$ a simple formula for the value of the Hilbert symbol of degree $\ell$ is not known. Nevertheless, in this case there also exists a $\mathfrak{p}$-adic unit $u_{\mathfrak{p}}$ satisfying 3.3 (see [1, Example 2.12]).

In both cases the element $u_{\mathfrak{p}}$ is $\mathfrak{p}$-primary, i.e. the extension $K_{\mathfrak{p}}\left(\sqrt[\ell]{u_{\mathfrak{p}}}\right) / K_{\mathfrak{p}}$ is unramified. We call $u_{\mathfrak{p}}$ the $\mathfrak{p}$-adic primary unit normalized with respect to $\zeta$ and denote by $u_{\mathfrak{p}}(\zeta)$ or just $u_{\mathfrak{p}}$ if $\zeta$ is fixed. Hilbert symbol is nondegenerate, so for a fixed $\zeta$ the element $u_{\mathfrak{p}}(\zeta)$ is uniquely determined up to $\ell$ th power in the field $K_{\mathfrak{p}}$.

For primes $\mathfrak{p}$ and $\mathfrak{p}^{\prime}$ of $K$ a local isomorphism $\varphi: \dot{K}_{\mathfrak{p}} / \dot{K}_{\mathfrak{p}}^{\ell} \rightarrow \dot{K}_{\mathfrak{p}^{\prime}} / \dot{K}_{\mathfrak{p}^{\prime}}^{\ell}$ is said to preserve Hilbert symbols, if

$$
(x, y)_{\mathfrak{p}}=(\varphi(x), \varphi(y))_{\mathfrak{p}^{\prime}} \quad \text { for all } x, y \in \dot{K}_{\mathfrak{p}}
$$

If $\mathfrak{p}$ and $\mathfrak{p}^{\prime}$ are finite primes and

$$
\operatorname{ord}_{\mathfrak{p}} x \equiv \operatorname{ord}_{\mathfrak{p}^{\prime}} \varphi(x)(\bmod \ell) \quad \text { for every } x \in \dot{K}_{\mathfrak{p}} / \dot{K}_{\mathfrak{p}}^{\ell},
$$

then the isomorphism $\varphi$ is called tame. Otherwise $\varphi$ is called wild.

There exists a relatively easy necessary and sufficient condition for the existence of a local isomorphism preserving Hilbert symbols (cf. [3, Lemma 2.2]). 
Proposition 3.4. Suppose $\mathfrak{p}$ and $\mathfrak{p}^{\prime}$ are finite primes of the field $K$. There exists a local isomorphism $\dot{K}_{\mathfrak{p}} / \dot{K}_{\mathfrak{p}}^{\ell} \rightarrow \dot{K}_{\mathfrak{p}^{\prime}} / \dot{K}_{\mathfrak{p}^{\prime}}^{\ell}$ preserving Hilbert symbols if and only if $\mathrm{rk}_{\ell} \dot{K}_{\mathfrak{p}} / \dot{K}_{\mathfrak{p}}^{\ell}=\mathrm{rk}_{\ell} \dot{K}_{\mathfrak{p}^{\prime}} / \dot{K}_{\mathfrak{p}^{\prime}}^{\ell}$.

By [10, Ch.3, Prop.1.5]:

$$
\mathrm{rk}_{\ell} \dot{K}_{\mathfrak{p}} / \dot{K}_{\mathfrak{p}}^{\ell}= \begin{cases}0, & \text { if } \mathfrak{p} \text { is complex } \\ 1, & \text { if } \mathfrak{p} \text { is real }(\ell=2), \\ {\left[K_{\mathfrak{p}}: \mathbb{Q}_{\ell}\right]+2,} & \text { if } \mathfrak{p} \text { is } \ell \text {-adic, } \\ 2, & \text { in other cases. }\end{cases}
$$

As a consequence we get the following.

Proposition 3.5. Let $\mathfrak{p}$ and $\mathfrak{p}^{\prime}$ be primes of $K$. If there exists an isomorphism $\dot{K}_{\mathfrak{p}} / \dot{K}_{\mathfrak{p}}^{\ell} \rightarrow \dot{K}_{\mathfrak{p}^{\prime}} / \dot{K}_{\mathfrak{p}^{\prime}}^{\ell}$ preserving Hilbert symbols, then:

(i) $\mathfrak{p}$ is finite $\Leftrightarrow \mathfrak{p}^{\prime}$ is finite.

(ii) $\mathfrak{p}$ is $\ell$-adic $\Leftrightarrow \mathfrak{p}^{\prime}$ is $\ell$-adic and $\left[K_{\mathfrak{p}}: \mathbb{Q}_{\ell}\right]=\left[K_{\mathfrak{p}^{\prime}}: \mathbb{Q}_{\ell}\right]$.

(iii) $\mathfrak{p}$ is real $\Leftrightarrow \mathfrak{p}^{\prime}$ is real.

Lemma 3.6. Let $\mathfrak{p}$ and $\mathfrak{p}^{\prime}$ be primes of $K$. The isomorphism $\varphi: \dot{K}_{\mathfrak{p}} / \dot{K}_{\mathfrak{p}}^{\ell} \rightarrow$ $\dot{K}_{\mathfrak{p}^{\prime}} / \dot{K}_{\mathfrak{p}^{\prime}}^{\ell}$ preserving Hilbert symbols is tame if and only if $\varphi\left(u_{\mathfrak{p}} \dot{K}_{\mathfrak{p}}^{\ell}\right)=u_{\mathfrak{p}^{\prime}} \dot{K}_{\mathfrak{p}^{\prime}}^{\ell}$.

Proof. Obviously, if $\varphi\left(u_{\mathfrak{p}} \dot{K}_{\mathfrak{p}}^{\ell}\right)=u_{\mathfrak{p}^{\prime}} \dot{K}_{\mathfrak{p}^{\prime}}^{\ell}$, then $\varphi$ is tame. Suppose that $\varphi$ preserves Hilbert symbols and is tame. Let $\varphi\left(u_{\mathfrak{p}} \dot{K}_{\mathfrak{p}}^{\ell}\right)=w \dot{K}_{\mathfrak{p}^{\prime}}^{\ell}$. Then

$$
\begin{aligned}
\left(u_{\mathfrak{p}^{\prime}}, \varphi(x)\right)_{\mathfrak{p}^{\prime}} & =\zeta^{\operatorname{ord}_{\mathfrak{p}^{\prime}} \varphi(x)}=\zeta^{\operatorname{ord}_{\mathfrak{p}} x} \\
& =\left(u_{\mathfrak{p}}, x\right)_{\mathfrak{p}}=(w, \varphi(x))_{\mathfrak{p}^{\prime}} \quad \text { for every } x \in \dot{K}_{\mathfrak{p}} .
\end{aligned}
$$

Since Hilbert symbol is nondegenerate, we have

$$
\varphi\left(u_{\mathfrak{p}} \dot{K}_{\mathfrak{p}}^{\ell}\right)=w \dot{K}_{\mathfrak{p}^{\prime}}^{\ell}=u_{\mathfrak{p}^{\prime}} \dot{K}_{\mathfrak{p}^{\prime}}^{\ell}
$$

Lemma 3.7. Let $\mathfrak{p}$ be a prime of $K$ and let $H, H^{\prime}$ be subgroups of $\dot{K}_{\mathfrak{p}} / \dot{K}_{\mathfrak{p}}^{\ell}$. Any group isomorphism $\tau: H \rightarrow H^{\prime}$ preserving Hilbert symbols can be extended to a group automorphism of $\dot{K}_{\mathfrak{p}} / \dot{K}_{\mathfrak{p}}^{\ell}$ preserving Hilbert symbols. Moreover, if $\tau$ is tame on $H$, i.e. $\operatorname{ord}_{\mathfrak{p}} a \equiv \operatorname{ord}_{\mathfrak{p}} \tau(a)(\bmod \ell)$ for every $a \in H$ and $\tau\left(u_{\mathfrak{p}} \dot{K}_{\mathfrak{p}}^{\ell}\right)=u_{\mathfrak{p}} \dot{K}_{\mathfrak{p}}^{\ell}$, when $u_{\mathfrak{p}} \dot{K}_{\mathfrak{p}}^{\ell} \in H$, then the isomorphism $\tau$ can be extended to a tame automorphism preserving Hilbert symbols. 
PROof. From the standard properties of Hilbert symbols, it follows that the mapping

$$
\beta_{\mathfrak{p}}: \dot{K}_{\mathfrak{p}} / \dot{K}_{\mathfrak{p}}^{\ell} \times \dot{K}_{\mathfrak{p}} / \dot{K}_{\mathfrak{p}}^{\ell} \rightarrow \mathbb{F}_{\ell}, \quad(x, y)_{\mathfrak{p}}=\zeta^{\beta_{\mathfrak{p}}(x, y)} \quad \text { for } x, y \in \dot{K}_{\mathfrak{p}} / \dot{K}_{\mathfrak{p}}^{\ell}
$$

is nondegenerate bilinear and antisymmetric, so it is also alternating. To get the statement one can use a standard prolongation theorem from bilinear algebra.

Lemma 3.8. Let $\mathfrak{r}$ be an $\ell$-adic prime of the field $K$. The linear mapping $\varphi: \dot{K}_{\mathfrak{r}} / \dot{K}_{\mathfrak{r}}^{\ell} \rightarrow \dot{K}_{\mathfrak{r}} / \dot{K}_{\mathfrak{r}}^{\ell}$ uniquely defined by $\varphi\left(u_{\mathfrak{r}} \dot{K}_{\mathfrak{r}}^{\ell}\right)=u_{\mathfrak{r}} \pi_{\mathfrak{r}} \dot{K}_{\mathfrak{r}}^{\ell}, \varphi\left(\pi_{\mathfrak{r}} \dot{K}_{\mathfrak{r}}^{\ell}\right)=$ $\pi_{\mathfrak{r}} \dot{K}_{\mathfrak{r}}^{\ell}$, and $\varphi\left(v \dot{K}_{\mathfrak{r}}^{\ell}\right)=v \dot{K}_{\mathfrak{r}}^{\ell}$ for every $v \dot{K}_{\mathfrak{r}}^{\ell} \in\left\langle u_{\mathfrak{r}} \dot{K}_{\mathfrak{r}}^{\ell}, \pi_{\mathfrak{r}} \dot{K}_{\mathfrak{r}}^{\ell}\right\rangle^{\perp}$ is an automorphism of the bilinear space $\left(\dot{K}_{\mathfrak{r}} / \dot{K}_{\mathfrak{r}}^{\ell}, \beta_{\mathfrak{r}}\right)$.

Proof. It is a routine matter to check that $\varphi$ is a group automorphism. Thus it suffices to show that it preserves Hilbert symbols. From properties of Hilbert symbol of degree $\ell$ it follows that $(x, x)_{\mathfrak{r}}=1$ for every $x \in \dot{K}_{\mathfrak{r}} / \dot{K}_{\mathfrak{r}}^{\ell}$. Moreover,

$$
\begin{aligned}
& \left(\pi_{\mathfrak{r}}, u_{\mathfrak{r}}\right)_{\mathfrak{r}}=\left(\pi_{\mathfrak{r}}, \pi_{\mathfrak{r}}\right)_{\mathfrak{r}}\left(\pi_{\mathfrak{r}}, u_{\mathfrak{r}}\right)_{\mathfrak{r}}=\left(\pi_{\mathfrak{r}}, \pi_{\mathfrak{r}} u_{\mathfrak{r}}\right)_{\mathfrak{r}}, \\
& \left(\pi_{\mathfrak{r}}, v\right)_{\mathfrak{r}}=1=\left(u_{\mathfrak{r}} \pi_{\mathfrak{r}}, v\right)_{\mathfrak{r}} \quad \text { for every } v \dot{K}_{\mathfrak{r}}^{\ell} \in\left\langle u_{\mathfrak{r}} \dot{K}_{\mathfrak{r}}^{\ell}, \pi_{\mathfrak{r}} \dot{K}_{\mathfrak{r}}^{\ell}\right\rangle^{\perp}, \\
& \left(u_{\mathfrak{r}}, v\right)_{\mathfrak{r}}=1=\left(u_{\mathfrak{r}} \pi_{\mathfrak{r}}, v\right)_{\mathfrak{r}} \quad \text { for every } v \dot{K}_{\mathfrak{r}}^{\ell} \in\left\langle u_{\mathfrak{r}} \dot{K}_{\mathfrak{r}}^{\ell}, \pi_{\mathfrak{r}} \dot{K}_{\mathfrak{r}}^{\ell}\right\rangle^{\perp}
\end{aligned}
$$

Lemma 3.9. Let $\mathfrak{p}, \mathfrak{q}$ be non- $\ell$-adic finite primes of $K$. The linear mapping $\varphi: \dot{K}_{\mathfrak{p}} / \dot{K}_{\mathfrak{p}}^{\ell} \rightarrow \dot{K}_{\mathfrak{q}} / \dot{K}_{\mathfrak{q}}^{\ell}$ uniquely defined by $\varphi\left(u_{\mathfrak{p}} \dot{K}_{\mathfrak{p}}^{\ell}\right)=u_{\mathfrak{q}} \pi_{\mathfrak{q}} \dot{K}_{\mathfrak{q}}^{\ell}, \quad \varphi\left(\pi_{\mathfrak{p}} \dot{K}_{\mathfrak{p}}^{\ell}\right)=$ $\pi_{\mathfrak{q}} \dot{K}_{\mathfrak{q}}^{\ell}$ is an isomorphism of the bilinear spaces $\left(\dot{K}_{\mathfrak{p}} / \dot{K}_{\mathfrak{p}}^{\ell}, \beta_{\mathfrak{p}}\right)$ and $\left(\dot{K}_{\mathfrak{q}} / \dot{K}_{\mathfrak{q}}^{\ell}, \beta_{\mathfrak{q}}\right)$.

Proof. Analogously as in the previous lemma.

REMARK 3.10. If $(T, t)$ is a Hilbert self-equivalence of degree $\ell$ of $K$, then for any $\mathfrak{p} \in \Omega(K)$ the isomorphism $t$ induces a local group isomorphism $t_{\mathfrak{p}}: \dot{K}_{\mathfrak{p}} / \dot{K}_{\mathfrak{p}}^{\ell} \rightarrow \dot{K}_{T \mathfrak{p}} / \dot{K}_{T \mathfrak{p}}^{\ell}$ preserving Hilbert symbols of degree $\ell$ (see [2, Lemma 2.3]). Notice that $t_{\mathfrak{p}}$ is tame if and only if $\mathfrak{p}$ is a tame prime of the self-equivalence $(T, t)$.

Assume that $(T, t)$ is a self-equivalence and $S$ is a Hasse set of primes of the field $K$. The group $I_{S}$ of $S$-ideals of $K$ is a free group generated by the set of all prime ideals and $T$ maps finite primes to finite primes, so the bijection $T: \Omega(K) \rightarrow \Omega(K)$ induces a group automorphism $\widetilde{T}: I_{S} \rightarrow I_{S}$ such that $\widetilde{T}\left(\prod_{\mathfrak{p} \notin S} \mathfrak{p}^{e_{\mathfrak{p}}}\right)=\prod_{\mathfrak{p} \notin S} T(\mathfrak{p})^{e_{\mathfrak{p}}}$. 
Proposition 3.11. Assume that the Hilbert self-equivalence $(T, t)$ is tame outside a Hasse set $S$. The above defined isomorphism $\widetilde{T}: I_{S} \rightarrow I_{S}$ induces a group automorphism $\widehat{T}$ of the group $C_{S} / C_{S}^{\ell}$ such that $\widehat{T}\left(\operatorname{clp} C_{S}^{\ell}\right)=\operatorname{cl} T(\mathfrak{p}) C_{S}^{\ell}$ for every prime $\mathfrak{p} \notin S$.

Proof. For any $S$-ideals $\mathfrak{a}, \mathfrak{b}$ of $K$, we have

$$
\operatorname{cl} \mathfrak{a} C_{S}^{\ell}=\operatorname{cl} \mathfrak{b} C_{S}^{\ell} \Longleftrightarrow x \mathcal{O}_{S} \cdot \mathfrak{a}=\mathfrak{b} \cdot \mathfrak{J}^{\ell} \quad \text { for some } x \in \dot{K}, \mathfrak{J} \in I_{S},
$$

that is,

$$
\operatorname{cl} \mathfrak{a} C_{S}^{\ell}=\operatorname{cl} \mathfrak{b} C_{S}^{\ell} \Longleftrightarrow \exists_{x \in \dot{K}} \forall_{\mathfrak{p} \notin S} \quad \operatorname{ord}_{\mathfrak{p}} x+\operatorname{ord}_{\mathfrak{p}} \mathfrak{a} \equiv \operatorname{ord}_{\mathfrak{p}} \mathfrak{b} \quad(\bmod \ell) .
$$

From the condition

$$
\operatorname{ord}_{\mathfrak{p}} x \equiv \operatorname{ord}_{T \mathfrak{p}} t x \quad(\bmod \ell) \quad \text { for every } \quad x \in \dot{K} / \dot{K}^{\ell} \mathfrak{p} \in \Omega(K) \backslash S
$$

we get a sequence of equivalences

$$
\begin{aligned}
& \operatorname{cl} \mathfrak{a} C_{S}^{\ell}=\operatorname{cl} \mathfrak{b} C_{S}^{\ell} \Longleftrightarrow \exists_{x \in \dot{K}} \forall_{\mathfrak{p} \notin S} \quad \operatorname{ord}_{\mathfrak{p}} x+\operatorname{ord}_{\mathfrak{p}} \mathfrak{a} \equiv \operatorname{ord}_{\mathfrak{p}} \mathfrak{b} \quad(\bmod \ell) \\
& \Longleftrightarrow t(x)+\operatorname{ord}_{T \mathfrak{p}} \widetilde{T}(\mathfrak{a}) \equiv \operatorname{ord}_{T \mathfrak{p}} \widetilde{T}(\mathfrak{b})(\bmod \ell) \Longleftrightarrow \operatorname{cl} \widetilde{T}(\mathfrak{a}) C_{S}^{\ell}=\operatorname{cl} \widetilde{T}(\mathfrak{b}) C_{S}^{\ell},
\end{aligned}
$$

which assures us that $\widehat{T}$ is a well defined injection. It is easy to check that $\widehat{T}$ is a group automorphism of $C_{S} / C_{S}^{\ell}$.

Let $S$ and $S^{\prime}$ be sufficiently large Hasse sets of $K$. A triple $\left(T_{S}, t_{S}, \prod_{\mathfrak{p} \in S} t_{\mathfrak{p}}\right)$ is called a small $S$-equivalence of $K$ if

$-T_{S}: S \rightarrow S^{\prime}$ is a bijection,

- $t_{S}: E_{S} / \dot{K}^{\ell} \rightarrow E_{S^{\prime}} / \dot{K}^{\ell}$ is a group isomorphism,

- for any $\mathfrak{p} \in S$ the mapping $t_{\mathfrak{p}}: \dot{K}_{\mathfrak{p}} / \dot{K}_{\mathfrak{p}}^{\ell} \rightarrow \dot{K}_{T \mathfrak{p}} / \dot{K}_{T \mathfrak{p}}^{\ell}$ is a group isomorphism preserving the symbol $(\cdot, \cdot)_{\mathfrak{p}}$ of degree $\ell$, i.e.

$$
(x, y)_{\mathfrak{p}}=\left(t_{\mathfrak{p}} x, t_{\mathfrak{p}} y\right)_{T \mathfrak{p}} \quad \text { for all } x, y \in \dot{K}_{\mathfrak{p}} / \dot{K}_{\mathfrak{p}}^{\ell},
$$

- the following diagram

$$
\begin{array}{ccc}
E_{S} / \dot{K}^{\ell} \stackrel{i_{S}}{\longrightarrow} & \prod_{\mathfrak{p} \in S} \dot{K}_{\mathfrak{p}} / \dot{K}_{\mathfrak{p}}^{\ell} \\
\downarrow t_{S} & & \\
E_{S^{\prime}} / \dot{K}^{\ell} & \stackrel{i_{S^{\prime}}}{\longrightarrow} & \prod_{\mathfrak{p} \in S} \dot{K}_{T \mathfrak{p}} / \dot{K}_{T \mathfrak{p}}^{\ell}
\end{array}
$$

commutes, i.e. $t_{S}\left(x \dot{K}^{\ell}\right) \equiv t_{\mathfrak{p}}\left(x \dot{K}_{\mathfrak{p}}^{\ell}\right) \bmod \dot{K}_{T \mathfrak{p}}^{\ell}$ for every $\mathfrak{p} \in S, x \in E_{S}$. 
The following theorem is a special case of [3, Theorem 3.4].

TheOrem 3.12. Any small $S$-equivalence $\left(T_{S}, t_{S}, \prod_{\mathfrak{p} \in S} t_{\mathfrak{p}}\right)$ of degree $\ell$ of the field $K$ can be extended to a self-equivalence $(T, t)$ of $K$, which is tame outside $S$, i.e.

$$
\mathfrak{p} \notin \mathcal{W}(T, t) \quad \text { for every } \mathfrak{p} \in \Omega(K) \backslash S .
$$

\section{Hilbert self-equivalence with one wild prime}

In this section for a field satisfying $(\mathrm{C} 1),(\mathrm{C} 2)$ and a finite prime $\mathfrak{p}$, the class of which is an $\ell$ th power in the ideal class group, we shall construct a Hilbert self-equivalence with $\mathfrak{p}$ as the unique wild prime.

As we have already noticed, in the case of a field satisfying $(\mathrm{C} 1)$ and $(\mathrm{C} 2)$ every infinite prime is complex. Therefore its Hilbert symbol is trivial and in constructions of self-equivalences we may ignore infinite primes.

THEOREM 4.1. If $K$ is a number field satisfying $(\mathrm{C} 1),(\mathrm{C} 2)$ and $\mathfrak{p}$ is a finite prime of $K$ such that $\operatorname{cl} \mathfrak{p} \in C_{K}^{\ell}$, then there exists a Hilbert self-equivalence $(T, t)$ of degree $\ell$ of $K$ such that $\mathcal{W}(T, t)=\{\mathfrak{p}\}$.

Proof. Let $\zeta \in K$ be a fixed primitive $\ell$ th root of unity and let $n=$ $\mathrm{rk}_{\ell} C_{K}$.

Denote by $S_{0}$ the subset of $\Omega(K)$ consisting of all infinite primes and the unique $\ell$-adic prime $\mathfrak{r}$. Thus $C_{K} / C_{K}^{\ell}=C_{S_{0}} / C_{S_{0}}^{\ell}$, because cl $\mathfrak{r} \in C_{K}^{\ell}$. Moreover, there exists $x_{\mathfrak{r}} \in \dot{K}$ and a fractional ideal $\mathfrak{J}$ of $K$ such that $\left(x_{\mathfrak{r}}\right)=\mathfrak{r} \cdot \mathfrak{J}^{\ell}$. Of course, $x_{\mathfrak{r}} \in E_{S_{0}}$ and $\mathrm{rk}_{\ell} E_{S_{0}} / \dot{K}^{\ell}=\mathrm{rk}_{\ell} E_{K} / \dot{K}^{\ell}+1$.

Choose primes $\mathfrak{p}_{1}, \ldots, \mathfrak{p}_{n}$ of $K$ such that the classes $c l \mathfrak{p}_{1}, \ldots, c l \mathfrak{p}_{n}$ form a basis of $C_{K} / C_{K}^{\ell}$. For $S=S_{0} \cup\left\{\mathfrak{p}_{1}, \ldots, \mathfrak{p}_{n}\right\}$ we have $\mathrm{rk}_{\ell} C_{S}=0$ (i.e. $S$ is sufficiently large) and $E_{S} / \dot{K}^{\ell}=E_{S_{0}} / \dot{K}^{\ell}$.

By Lemma 2.4 there exist $b_{1}, \ldots, b_{n} \in \Delta_{S_{0}}$ such that

$$
\left(\frac{b_{i}}{\mathfrak{p}_{i}}\right)_{\ell}=\zeta, \quad\left(\frac{b_{i}}{\mathfrak{p}_{j}}\right)_{\ell}=1 \quad \text { for all } i, j \in\{1, \ldots, n\}, i \neq j .
$$

Multiplying $x_{\mathfrak{r}}$, if necessary, by appropriate powers of $b_{i}, i \in\{1, \ldots, n\}$, we may assume that

$$
\left(\frac{x_{\mathfrak{r}}}{\mathfrak{p}_{i}}\right)_{\ell}=1 \text { for all } i \in\{1, \ldots, n\}
$$


which gives $x_{\mathfrak{r}} \in \dot{K}_{\mathfrak{p}_{i}}^{\ell}$ for $i=1, \ldots, n$. By Corollary 2.5 we obtain

$$
\begin{aligned}
E_{S} / \dot{K}^{\ell} & =E_{S_{0}} / \dot{K}^{\ell}=U_{S_{0}} \dot{K}^{\ell} / \dot{K}^{\ell} \oplus \Delta_{S_{0}} / \dot{K}^{\ell} \\
& =U_{K} \dot{K}^{\ell} / \dot{K}^{\ell} \oplus\left\langle x_{\mathfrak{r}} \dot{K}^{\ell}\right\rangle \oplus\left\langle b_{1} \dot{K}^{\ell}, \ldots, b_{n} \dot{K}^{\ell}\right\rangle .
\end{aligned}
$$

We shall proceed dividing the proof into two cases.

(I) Assume that $\mathfrak{p}$ is $\ell$-adic, that is, $\mathfrak{p}=\mathfrak{r}$.

Notice that $\left(y, x_{\mathfrak{r}}\right)_{\mathfrak{v}}=1$ for every $y \in E_{S}$ and every prime $\mathfrak{v} \neq \mathfrak{r}$, because $y$ and $x_{\mathfrak{r}}$ are $\mathfrak{v}$-adic units modulo $\dot{K}_{\mathfrak{v}}^{\ell}$. Hence, by Hilbert reciprocity law, $\left(y, x_{\mathfrak{r}}\right)_{\mathfrak{r}}=1$ for every $y \in E_{S}$. In particular the class $u_{\mathfrak{r}} \dot{K}_{\mathfrak{r}}^{\ell}$ of the primary $\mathfrak{r}$-adic unit $u_{\mathfrak{r}}$ does not belong to the group $E_{S} \dot{K}_{\mathfrak{r}}^{\ell} / \dot{K}_{\mathfrak{r}}^{\ell}$. Thus we clearly have the inclusion

$$
E_{S} \dot{K}_{\mathfrak{r}}^{\ell} / \dot{K}_{\mathfrak{r}}^{\ell} \subseteq\left\langle\pi_{\mathfrak{r}} \dot{K}_{\mathfrak{r}}^{\ell}\right\rangle \oplus\left\langle u_{\mathfrak{r}} \dot{K}_{\mathfrak{r}}^{\ell}, \pi_{\mathfrak{r}} \dot{K}_{\mathfrak{r}}^{\ell}\right\rangle^{\perp}
$$

in the bilinear space $\left(\dot{K}_{\mathfrak{r}} / \dot{K}_{\mathfrak{r}}^{\ell}, \beta_{\mathfrak{r}}\right)$, where $\pi_{\mathfrak{r}} \in \dot{K}_{\mathfrak{r}}$ is a uniformizer such that $\pi_{\mathfrak{r}} \equiv x_{\mathfrak{r}} \bmod \dot{K}_{\mathfrak{r}}^{\ell}$.

Let us define the triple $\left(T_{S}, t_{S}, \prod_{\mathfrak{v} \in S} t_{\mathfrak{v}}\right)$ as follows

$$
\begin{array}{ll}
T_{S}: S \rightarrow S, & T_{S}=\mathrm{id}_{S}, \\
t_{S}: E_{S} / \dot{K}^{\ell} \rightarrow E_{S} / \dot{K}^{\ell}, & t_{S}=\mathrm{id}_{E_{S} / \dot{K}^{\ell}} \\
t_{\mathfrak{v}}: \dot{K}_{\mathfrak{v}} / \dot{K}_{\mathfrak{v}}^{\ell} \rightarrow \dot{K}_{\mathfrak{v}} / \dot{K}_{\mathfrak{v}}^{\ell}, & t_{\mathfrak{v}}=\mathrm{id}_{\dot{K}_{\mathfrak{v}} / \dot{K}_{\mathfrak{v}}^{\ell}} \quad \text { for every } \mathfrak{v} \in S \backslash\{\mathfrak{r}\}, \\
t_{\mathfrak{r}}: \dot{K}_{\mathfrak{r}} / \dot{K}_{\mathfrak{r}}^{\ell} \rightarrow \dot{K}_{\mathfrak{r}} / \dot{K}_{\mathfrak{r}}^{\ell}, & t_{\mathfrak{r}}\left(u_{\mathfrak{r}} \dot{K}_{\mathfrak{r}}^{\ell}\right)=u_{\mathfrak{r}} \pi_{\mathfrak{r}} \dot{K}_{\mathfrak{r}}^{\ell}, t_{\mathfrak{r}}\left(\pi_{\mathfrak{r}} \dot{K}_{\mathfrak{r}}^{\ell}\right)=\pi_{\mathfrak{r}} \dot{K}_{\mathfrak{r}}^{\ell}, \text { and } \\
& t_{\mathfrak{r}}\left(v \dot{K}_{\mathfrak{r}}^{\ell}\right)=v \dot{K}_{\mathfrak{r}}^{\ell} \text { for every } v \dot{K}_{\mathfrak{r}}^{\ell} \in\left\langle u_{\mathfrak{r}} \dot{K}_{\mathfrak{r}}^{\ell}, \pi_{\mathfrak{r}} \dot{K}_{\mathfrak{r}}^{\ell}\right\rangle^{\perp} .
\end{array}
$$

Every isomorphism $t_{\mathfrak{v}}$ for $\mathfrak{v} \in S$ as well as $t_{\mathfrak{r}}$, by Lemma 3.8, preserve Hilbert symbols.

We have the following equivalences

$$
t_{S}\left(y \dot{K}^{\ell}\right) \equiv t_{\mathfrak{v}}\left(y \dot{K}_{\mathfrak{v}}^{\ell}\right) \bmod \dot{K}_{\mathfrak{v}}^{\ell} \quad \text { for all } y \in E_{S}, \mathfrak{v} \in S
$$

Indeed, for $\mathfrak{v} \neq \mathfrak{r}$ it follows directly from the definitions of $t_{S}$ and local isomorphisms, whereas for $\mathfrak{v}=\mathfrak{r}$, by 4.1 , we have $t_{\mathfrak{r}}\left(z \dot{K}_{\mathfrak{v}}^{\ell}\right)=z \dot{K}_{\mathfrak{v}}^{\ell} \equiv t_{S}\left(z \dot{K}^{\ell}\right) \bmod$ $\dot{K}_{\mathfrak{v}}^{\ell}$ for every $z \in E_{S}$.

Thus, we showed that

$$
\prod_{\mathfrak{v} \in S} t_{\mathfrak{v}} \circ i_{S}=i_{S} \circ t_{S}
$$


and $\left(T_{S}, t_{S}, \prod_{\mathfrak{v} \in S} t_{\mathfrak{v}}\right)$ is a small $S$-equivalence. By Theorem 3.12 , it can be extended to a Hilbert self-equivalence $(T, t)$, which is tame outside $S$. Notice that the prime $\mathfrak{r}$ is the only prime in $S$, for which the local isomorphism $t_{\mathfrak{r}}$ is not tame. Hence $\mathcal{W}(T, t)=\{\mathfrak{r}\}$.

(II) Suppose $\mathfrak{p} \neq \mathfrak{r}$.

Since the class $\mathrm{cl} \mathfrak{p}$ is an $\ell$ th power in the group $C_{K}$, there exist a fractional ideal $\mathfrak{J}_{1}$ and $x_{\mathfrak{p}} \in \dot{K}$ such that $\left(x_{\mathfrak{p}}\right)=\mathfrak{p} \cdot \mathfrak{J}_{1}^{\ell}$. Let $S_{1}=S \cup\{\mathfrak{p}\}$. Obviously, $x_{\mathfrak{p}} \in E_{S_{1}}, \quad \mathrm{rk}_{\ell} C_{S_{1}}=0$ and $\mathrm{rk}_{\ell} E_{S_{1}} / \dot{K}^{\ell}=\mathrm{rk}_{\ell} E_{S} / \dot{K}^{\ell}+1$. This gives the following decomposition

$$
\begin{aligned}
E_{S_{1}} / \dot{K}^{\ell} & =E_{S} / \dot{K}^{\ell} \oplus\left\langle x_{\mathfrak{p}} \dot{K}^{\ell}\right\rangle \\
& =U_{K} \dot{K}^{\ell} / \dot{K}^{\ell} \oplus\left\langle b_{1} \dot{K}^{\ell}, \ldots, b_{n} \dot{K}^{\ell}\right\rangle \oplus\left\langle x_{\mathfrak{r}} \dot{K}^{\ell}\right\rangle \oplus\left\langle x_{\mathfrak{p}} \dot{K}^{\ell}\right\rangle .
\end{aligned}
$$

Now consider two subcases.

(II.1) Assume $\left(\frac{y}{\mathfrak{p}}\right)_{\ell}=1$ for every $y \in E_{S}$.

It follows that

$$
E_{S} \dot{K}_{\mathfrak{p}}^{\ell} / \dot{K}_{\mathfrak{p}}^{\ell}=\left\{\dot{K}_{\mathfrak{p}}^{\ell}\right\}
$$

Let us take a $\mathfrak{p}$-adic uniformizer $\pi_{\mathfrak{p}}$ such that $\pi_{\mathfrak{p}} \equiv x_{\mathfrak{p}} \bmod \dot{K}_{\mathfrak{p}}^{\ell}$ and define the triple $\left(T_{S_{1}}, t_{S_{1}}, \prod_{\mathfrak{v} \in S_{1}} t_{\mathfrak{v}}\right)$ as follows

$$
\begin{array}{ll}
T_{S_{1}}: S_{1} \rightarrow S_{1}, & T_{S_{1}}=\mathrm{id}_{S_{1}}, \\
t_{S_{1}}: E_{S_{1}} / \dot{K}^{\ell} \rightarrow E_{S_{1}} / \dot{K}^{\ell}, & t_{S_{1}}=\mathrm{id}_{E_{S_{1}} / \dot{K}^{\ell}}, \\
t_{\mathfrak{v}}: \dot{K}_{\mathfrak{v}} / \dot{K}_{\mathfrak{v}}^{\ell} \rightarrow \dot{K}_{\mathfrak{v}} / \dot{K}_{\mathfrak{v}}^{\ell}, & t_{\mathfrak{v}}=\mathrm{id}_{\dot{K}_{\mathfrak{v}} / \dot{K}_{\mathfrak{v}}^{\ell}} \quad \text { for every } \mathfrak{v} \in S, \\
t_{\mathfrak{p}}: \dot{K}_{\mathfrak{p}} / \dot{K}_{\mathfrak{p}}^{\ell} \rightarrow \dot{K}_{\mathfrak{p}} / \dot{K}_{\mathfrak{p}}^{\ell}, & t_{\mathfrak{p}}\left(u_{\mathfrak{p}} \dot{K}_{\mathfrak{p}}^{\ell}\right)=u_{\mathfrak{p}} \pi_{\mathfrak{p}} \dot{K}_{\mathfrak{p}}^{\ell}, \quad t_{\mathfrak{p}}\left(\pi_{\mathfrak{p}} \dot{K}_{\mathfrak{p}}^{\ell}\right)=\pi_{\mathfrak{p}} \dot{K}_{\mathfrak{p}}^{\ell} .
\end{array}
$$

The isomorphism $t_{\mathfrak{v}}$ for $\mathfrak{v} \in S$ as well as $t_{\mathfrak{p}}$, by Lemma 3.9, preserve Hilbert symbols. By $4.2, y \equiv 1 \bmod \dot{K}_{\mathfrak{p}}^{\ell}$ for every $y \in E_{S}$, so

$$
t_{\mathfrak{p}}\left(y \dot{K}_{\mathfrak{p}}^{\ell}\right)=1 \dot{K}_{\mathfrak{p}}^{\ell} \equiv y \dot{K}_{\mathfrak{p}}^{\ell} \equiv t_{S_{1}}\left(y \dot{K}^{\ell}\right) \bmod \dot{K}_{\mathfrak{p}}^{\ell} .
$$

Moreover,

$$
t_{\mathfrak{p}}\left(x_{\mathfrak{p}} \dot{K}_{\mathfrak{p}}^{\ell}\right)=t_{\mathfrak{p}}\left(\pi_{\mathfrak{p}} \dot{K}_{\mathfrak{p}}^{\ell}\right)=\pi_{\mathfrak{p}} \dot{K}_{\mathfrak{p}}^{\ell} \equiv x_{\mathfrak{p}} \dot{K}^{\ell} \equiv t_{S_{1}}\left(x_{\mathfrak{p}} \dot{K}^{\ell}\right) \bmod \dot{K}_{\mathfrak{p}}^{\ell} .
$$

Directly from the definition

$$
t_{\mathfrak{v}}\left(y \dot{K}_{\mathfrak{v}}^{\ell}\right)=y \dot{K}_{\mathfrak{v}}^{\ell} \equiv t_{S_{1}}\left(y \dot{K}^{\ell}\right) \bmod \dot{K}_{\mathfrak{v}}^{\ell}
$$


for every $y \in E_{S_{1}}$ and $\mathfrak{v} \in S$. Thus $\left(T_{S_{1}}, t_{S_{1}}, \prod_{\mathfrak{v} \in S_{1}} t_{\mathfrak{v}}\right)$ is a small $S_{1}$-equivalence of $K$. In the same way as in the previous case we conclude that there exists a self-equivalence $(T, t)$, for which $\mathfrak{p}$ is the only wild prime.

(II.2) Assume $\left(\frac{c}{\mathfrak{p}}\right)_{\ell} \neq 1$ for some $c \in E_{S}$.

Replacing $c$ by its power, we may assume $\left(\frac{c}{\mathfrak{p}}\right)_{\ell}=\zeta$, which follows $c \equiv$ $u_{\mathfrak{p}} \bmod \dot{K}_{\mathfrak{p}}^{\ell}$ and $\left(c, x_{\mathfrak{p}}\right)_{\mathfrak{p}}=\zeta$. Let $V$ be a direct complement of the subgroup $\left\langle c \dot{K}^{\ell}\right\rangle$ to the group $E_{S} / \dot{K}^{\ell}$, i.e.

$$
E_{S} / \dot{K}^{\ell}=V \oplus\left\langle c \dot{K}^{\ell}\right\rangle
$$

Multiplying, if needed, the elements of the group $V$ by appropriate powers of $c$ we may assume that $\left(\frac{z}{\mathfrak{p}}\right)_{\ell}=1$ for every $z \in V$, and then $V \subseteq \dot{K}_{\mathfrak{p}}^{\ell}$. Thus

$$
E_{S_{1}} / \dot{K}^{\ell}=V \oplus\left\langle c \dot{K}^{\ell}\right\rangle \oplus\left\langle x_{\mathfrak{p}} \dot{K}^{\ell}\right\rangle
$$

By [8, Lemma 2.1], there exists $x_{\mathfrak{q}} \in \dot{K}$ and $\mathfrak{q} \notin S$ such that

$$
\begin{array}{ll}
x_{\mathfrak{q}} \in \dot{K}_{\mathfrak{v}}^{\ell} & \text { for every } \mathfrak{v} \in S \backslash\{\mathfrak{r}\}, \\
x_{\mathfrak{q}} \equiv x_{\mathfrak{p}} \bmod \dot{K}_{\mathfrak{r}}^{\ell}, & \\
\operatorname{ord}_{\mathfrak{q}} x_{\mathfrak{q}}=1, & \\
\operatorname{ord}_{\mathfrak{v}} x_{\mathfrak{q}} \equiv 0 \quad(\bmod \ell) & \text { for every } \mathfrak{v} \in \Omega(K) \backslash(S \cup\{\mathfrak{q}\}) .
\end{array}
$$

Fix a $\mathfrak{q}$-adic uniformizer $\pi_{\mathfrak{q}} \equiv x_{\mathfrak{q}} \bmod \dot{K}_{\mathfrak{q}}^{\ell}$. The set $S_{1}^{\prime}=S \cup\{\mathfrak{q}\}$ is sufficiently large and we have

$$
E_{S_{1}^{\prime}} / \dot{K}^{\ell}=E_{S} / \dot{K}^{\ell} \oplus\left\langle x_{\mathfrak{q}} \dot{K}^{\ell}\right\rangle=V \oplus\left\langle c \dot{K}^{\ell}\right\rangle \oplus\left\langle x_{\mathfrak{q}} \dot{K}^{\ell}\right\rangle .
$$

Define $\left(T_{S_{1}}, t_{S_{1}}, \prod_{\mathfrak{v} \in S_{1}} t_{\mathfrak{v}}\right)$ as follows

$$
\begin{array}{ll}
T_{S_{1}}: S_{1} \rightarrow S_{1}^{\prime}, & \left.T_{S_{1}}\right|_{S}=\mathrm{id}_{S}, T_{S_{1}}(\mathfrak{p})=\mathfrak{q}, \\
t_{S_{1}}: E_{S_{1}} / \dot{K}^{\ell} \rightarrow E_{S_{1}^{\prime}} / \dot{K}^{\ell}, & \left.t_{S_{1}}\right|_{V}=\operatorname{id}_{V}, t_{S_{1}}\left(c \dot{K}^{\ell}\right)=c x_{\mathfrak{q}} \dot{K}^{\ell}, \\
& t_{S_{1}}\left(x_{\mathfrak{p}} \dot{K}^{\ell}\right)=x_{\mathfrak{q}} \dot{K}^{\ell}, \\
t_{\mathfrak{v}}: \dot{K}_{\mathfrak{v}} / \dot{K}_{\mathfrak{v}}^{\ell} \rightarrow \dot{K}_{\mathfrak{v}} / \dot{K}_{\mathfrak{v}}^{\ell}, & t_{\mathfrak{v}}=\operatorname{id}_{\dot{K}_{\mathfrak{v}} / \dot{K}_{\mathfrak{v}}^{\ell}} \quad \text { for every } \mathfrak{v} \in S_{1} \backslash\{\mathfrak{r}, \mathfrak{p}\}, \\
t_{\mathfrak{p}}: \dot{K}_{\mathfrak{p}} / \dot{K}_{\mathfrak{p}}^{\ell} \rightarrow \dot{K}_{\mathfrak{q}} / \dot{K}_{\mathfrak{q}}^{\ell}, & t_{\mathfrak{p}}\left(u_{\mathfrak{p}} \dot{K}_{\mathfrak{p}}^{\ell}\right)=u_{\mathfrak{q}} \pi_{\mathfrak{q}} \dot{K}_{\mathfrak{q}}^{\ell}, \quad t_{\mathfrak{p}}\left(\pi_{\mathfrak{p}} \dot{K}_{\mathfrak{p}}^{\ell}\right)=\pi_{\mathfrak{q}} \dot{K}_{\mathfrak{q}}^{\ell} .
\end{array}
$$


As in the previous cases we see that all defined above local isomorphisms preserve Hilbert symbols.

Definition of a local isomorphism $t_{\mathfrak{r}}: \dot{K}_{\mathfrak{r}} / \dot{K}_{\mathfrak{r}}^{\ell} \rightarrow \dot{K}_{\mathfrak{r}} / \dot{K}_{\mathfrak{r}}^{\ell}$ left. To define it we use Lemma 3.7. Consider subgroups $H=E_{S_{1}} \dot{K}_{\mathfrak{r}}^{\ell} / \dot{K}_{\mathfrak{r}}^{\ell}$ and $H^{\prime}=E_{S_{1}^{\prime}} \dot{K}_{\mathfrak{r}}^{\ell} / \dot{K}_{\mathfrak{r}}^{\ell}$ of $\dot{K}_{\mathfrak{r}} / \dot{K}_{\mathfrak{r}}^{\ell}$. We shall show that $t_{S_{1}}$ induces a tame isomorphism $H \rightarrow H^{\prime}$ preserving Hilbert symbols.

We start with checking that $t_{S_{1}}$ preserves the $\mathfrak{r}$-adic Hilbert symbol. It is obvious that $\left(t_{S_{1}}(y), t_{S_{1}}(z)\right)_{\mathfrak{r}}=(y, z)_{\mathfrak{r}}$ for $y, z \in V$. By the choice of $x_{\mathfrak{q}}$ we have $x_{\mathfrak{p}}=x_{\mathfrak{q}} \bmod \dot{K}_{\mathfrak{r}}^{\ell}$, so

$$
\left(y, x_{\mathfrak{p}}\right)_{\mathfrak{r}}=\left(y, x_{\mathfrak{q}}\right)_{\mathfrak{r}} \text { for every } y \in V
$$

and

$$
\left(c, x_{\mathfrak{p}}\right)_{\mathfrak{r}}=\left(c, x_{\mathfrak{q}}\right)_{\mathfrak{r}}=\left(c x_{\mathfrak{q}}, x_{\mathfrak{q}}\right)_{\mathfrak{r}} .
$$

Now notice that $(y, c)_{\mathfrak{v}}=1$ for every prime $\mathfrak{v} \neq \mathfrak{r}$, and then $(y, c)_{\mathfrak{r}}=1$ by Hilbert reciprocity law. By the choice of $x_{\mathfrak{p}}$ and $x_{\mathfrak{q}}$,

$$
\left(y, c x_{\mathfrak{q}}\right)_{\mathfrak{r}}=(y, c)_{\mathfrak{r}} \cdot\left(y, x_{\mathfrak{q}}\right)_{\mathfrak{r}}=(y, c)_{\mathfrak{r}} \cdot\left(y, x_{\mathfrak{p}}\right)_{\mathfrak{r}}=1=(y, c)_{\mathfrak{r}} \text { for } y \in V .
$$

Thus $t_{S_{1}}$ preserves the $\mathfrak{r}$-adic Hilbert symbol.

Now we shall show that $t_{S_{1}}$ induces a group isomorphism $\tau: H \rightarrow H^{\prime}$. To do this it suffices to explain that

$$
H=V \dot{K}_{\mathfrak{r}}^{\ell} \oplus\left\langle x_{\mathfrak{p}} \dot{K}_{\mathfrak{r}}^{\ell}\right\rangle \oplus\left\langle c \dot{K}_{\mathfrak{r}}^{\ell}\right\rangle, \quad H^{\prime}=V \dot{K}_{\mathfrak{r}}^{\ell} \oplus\left\langle x_{\mathfrak{q}} \dot{K}_{\mathfrak{r}}^{\ell}\right\rangle \oplus\left\langle c x_{\mathfrak{q}} \dot{K}_{\mathfrak{r}}^{\ell}\right\rangle .
$$

By the definition of $H$ and $H^{\prime}$,

$$
H=V \dot{K}_{\mathfrak{r}}^{\ell}+\left\langle x_{\mathfrak{p}} \dot{K}_{\mathfrak{r}}^{\ell}\right\rangle+\left\langle c \dot{K}_{\mathfrak{r}}^{\ell}\right\rangle, \quad H^{\prime}=V \dot{K}_{\mathfrak{r}}^{\ell}+\left\langle x_{\mathfrak{q}} \dot{K}_{\mathfrak{r}}^{\ell}\right\rangle+\left\langle c x_{\mathfrak{q}} \dot{K}_{\mathfrak{r}}^{\ell}\right\rangle,
$$

whereas by the choice of $x_{\mathfrak{q}}, V \dot{K}_{\mathfrak{r}}^{\ell} \oplus\left\langle x_{\mathfrak{p}} \dot{K}_{\mathfrak{r}}^{\ell}\right\rangle=V \dot{K}_{\mathfrak{r}}^{\ell} \oplus\left\langle x_{\mathfrak{q}} \dot{K}_{\mathfrak{r}}^{\ell}\right\rangle$.

On the one hand for every prime $\mathfrak{v} \notin\{\mathfrak{p}, \mathfrak{r}\}$ elements of $V \oplus\left\langle x_{\mathfrak{p}} \dot{K}^{\ell}\right\rangle$ are (modulo $\ell$ th powers) $\mathfrak{v}$-adic units, so $\left(y, x_{\mathfrak{p}}\right)_{\mathfrak{v}}=1$. Moreover, for $y=z x_{\mathfrak{p}}, z \in$ $V \subseteq \dot{K}_{\mathfrak{p}}^{\ell}$ we have $\left(y, x_{\mathfrak{p}}\right)_{\mathfrak{p}}=\left(z, x_{\mathfrak{p}}\right)_{\mathfrak{p}}\left(x_{\mathfrak{p}}, x_{\mathfrak{p}}\right)_{\mathfrak{p}}=1$. Thus $\left(y, x_{\mathfrak{p}}\right)_{\mathfrak{r}}=1$ by Hilbert reciprocity law and

$$
\left(y, x_{\mathfrak{p}}\right)_{\mathfrak{r}}=1, \forall y \in V \dot{K}_{\mathfrak{r}}^{\ell} \oplus\left\langle x_{\mathfrak{p}} \dot{K}_{\mathfrak{r}}^{\ell}\right\rangle .
$$

On the other hand $\left(c, x_{\mathfrak{p}}\right)_{\mathfrak{v}}=1$ for every $\mathfrak{v} \notin\{\mathfrak{p}, \mathfrak{r}\}$, because $c$ and $x_{\mathfrak{p}}$ are (modulo $\ell$ th powers) $\mathfrak{v}$-adic units. Thus, by Hilbert reciprocity law, $\left(c, x_{\mathfrak{p}}\right)_{\mathfrak{r}}=$ $\left(c, x_{\mathfrak{p}}\right)_{\mathfrak{p}}^{-1}=\zeta^{-1} \neq 1$. This fact together with 4.5 gives $c \dot{K}_{\mathfrak{r}}^{\ell} \notin V \dot{K}_{\mathfrak{r}}^{\ell} \oplus\left\langle x_{\mathfrak{p}} \dot{K}_{\mathfrak{r}}^{\ell}\right\rangle$ and explain the first part of 4.4 . 
To explain the second part of 4.4 observe that

$$
\left(c x_{\mathfrak{p}}, x_{\mathfrak{p}}\right)_{\mathfrak{r}}=\left(c, x_{\mathfrak{p}}\right)_{\mathfrak{r}} \cdot\left(x_{\mathfrak{p}}, x_{\mathfrak{p}}\right)_{\mathfrak{r}}=\zeta^{-1} \neq 1
$$

which with 4.5 gives $c x_{\mathfrak{p}} \dot{K}_{\mathfrak{r}}^{\ell} \notin V \dot{K}_{\mathfrak{r}}^{\ell} \oplus\left\langle x_{\mathfrak{q}} \dot{K}_{\mathfrak{r}}^{\ell}\right\rangle$.

As we notice above, the isomorphism $\tau$ preserves the $\mathfrak{r}$-adic Hilbert symbol. Notice else that

$$
\operatorname{ord}_{\mathfrak{r}} x_{\mathfrak{p}} \equiv 0 \equiv \operatorname{ord}_{\mathfrak{r}} x_{\mathfrak{q}} \quad(\bmod \ell) \quad \text { and } \quad \operatorname{ord}_{\mathfrak{r}} c \equiv \operatorname{ord}_{\mathfrak{r}} c x_{\mathfrak{q}} \quad(\bmod \ell)
$$

Hence $\tau$ is tame on $H$.

Now assume that the group $H$ contains the $\mathfrak{r}$-adic primary unit $u_{\mathfrak{r}}$. Then $u_{\mathfrak{r}} \dot{K}_{\mathfrak{r}}^{\ell}=v^{\epsilon_{1}} c^{\epsilon_{2}} x_{\mathfrak{p}}^{\epsilon_{3}} \dot{K}_{\mathfrak{r}}^{\ell}$ for some $\epsilon_{1}, \epsilon_{2}, \epsilon_{3} \in\{0,1, \ldots, \ell-1\}$. Since $x_{\mathfrak{p}}$ is an $\mathfrak{r}$-adic unit modulo $\dot{K}_{\mathfrak{r}}^{\ell},\left(u_{\mathfrak{r}}, x_{\mathfrak{p}}\right)_{\mathfrak{r}}=1$. Thus

$$
1=\left(v^{\epsilon_{1}} c^{\epsilon_{2}} x_{\mathfrak{p}}^{\epsilon_{3}}, x_{\mathfrak{p}}\right)_{\mathfrak{r}}=\left(v, x_{\mathfrak{p}}\right)_{\mathfrak{r}}^{\epsilon_{1}}\left(c, x_{\mathfrak{p}}\right)_{\mathfrak{r}}^{\epsilon_{2}}\left(x_{\mathfrak{p}}, x_{\mathfrak{p}}\right)_{\mathfrak{r}}^{\epsilon_{3}}=\left(c, x_{\mathfrak{p}}\right)_{\mathfrak{r}}^{\epsilon_{2}}=\zeta^{-\epsilon_{2}}
$$

Hence $\epsilon_{2}=0$ and $u_{\mathfrak{r}} \dot{K}_{\mathfrak{r}}^{\ell} \in V \dot{K}_{\mathfrak{r}}^{\ell} \oplus\left\langle x_{\mathfrak{p}} \dot{K}_{\mathfrak{r}}^{\ell}\right\rangle$. By the definition of $t_{S_{1}}$ we get $\tau\left(u_{\mathfrak{r}} \dot{K}_{\mathfrak{r}}^{\ell}\right)=u_{\mathfrak{r}} \dot{K}_{\mathfrak{r}}^{\ell}$.

Finally, all assumptions of Lemma 3.7 hold, so there exists a tame isomorphism $t_{\mathfrak{r}}: \dot{K}_{\mathfrak{r}} / \dot{K}_{\mathfrak{r}}^{\ell} \rightarrow \dot{K}_{\mathfrak{r}} / \dot{K}_{\mathfrak{r}}^{\ell}$, which is an extension of $\tau$ and preserves Hilbert symbols of degree $\ell$.

We shall show that $\left(T_{S_{1}}, t_{S_{1}}, \prod_{\mathfrak{v} \in S_{1}} t_{\mathfrak{v}}\right)$ is a small $S_{1}$-equivalence. To do this we should prove that the diagram

$$
\begin{array}{rr}
E_{S_{1}} / \dot{K}^{\ell} \stackrel{i_{S_{1}}}{\longrightarrow} & \prod_{\mathfrak{v} \in S_{1}} \dot{K}_{\mathfrak{v}} / \dot{K}_{\mathfrak{v}}^{\ell} \\
\downarrow t_{S_{1}} & \\
E_{S_{1}^{\prime}} / \dot{K}^{\ell} \stackrel{\prod_{\mathfrak{v} \in S_{1}} t_{\mathfrak{v}}}{\stackrel{i_{S_{1}^{\prime}}}{\longrightarrow}} & \prod_{\mathfrak{v} \in S_{1}} \dot{K}_{T \mathfrak{v}} / \dot{K}_{T \mathfrak{v}}^{\ell}
\end{array}
$$

commutes, i.e.

$$
t_{S_{1}}\left(x \dot{K}^{\ell}\right) \equiv t_{\mathfrak{v}}\left(x \dot{K}_{\mathfrak{v}}^{\ell}\right) \bmod \dot{K}_{T \mathfrak{v}}^{\ell}, \quad \text { for every } \mathfrak{v} \in S_{1}, x \in E_{S_{1}}
$$

Of course, $t_{S_{1}}\left(z \dot{K}^{\ell}\right) \equiv t_{\mathfrak{r}}\left(z \dot{K}_{\mathfrak{r}}^{\ell}\right) \bmod \dot{K}_{\mathfrak{r}}^{\ell}$ for every $z \in E_{S_{1}}$, by the definition of $t_{\mathfrak{r}}$.

Now concentrate on proving $t_{S_{1}}\left(z \dot{K}^{\ell}\right) \equiv t_{\mathfrak{p}}\left(z \dot{K}_{\mathfrak{p}}^{\ell}\right) \bmod \dot{K}_{\mathfrak{q}}^{\ell}$ for every $z \in$ $E_{S_{1}}$ It suffices to show this congruence for $x_{\mathfrak{p}}, c$ and $z \in V$.

By the definitions 4.3 ,

$$
t_{S_{1}}\left(x_{\mathfrak{p}} \dot{K}^{\ell}\right)=x_{\mathfrak{q}} \dot{K}^{\ell} \equiv \pi_{\mathfrak{q}} \dot{K}_{\mathfrak{q}}^{\ell}=t_{\mathfrak{p}}\left(\pi_{\mathfrak{p}} \dot{K}_{\mathfrak{p}}^{\ell}\right) \equiv t_{\mathfrak{p}}\left(x_{\mathfrak{p}} \dot{K}_{\mathfrak{p}}^{\ell}\right) \bmod \dot{K}_{\mathfrak{q}}^{\ell}
$$


Since the local isomorphisms preserve Hilbert symbols,

$$
\left(c, x_{\mathfrak{q}}\right)_{\mathfrak{q}}=\left(c, x_{\mathfrak{q}}\right)_{\mathfrak{q}}\left(x_{\mathfrak{q}}, x_{\mathfrak{q}}\right)_{\mathfrak{q}}=\left(c x_{\mathfrak{q}}, x_{\mathfrak{q}}\right)_{\mathfrak{q}}=\left(c, x_{\mathfrak{p}}\right)_{\mathfrak{p}}=\zeta,
$$

which means that $c=u_{\mathfrak{q}}$ is the primary $\mathfrak{q}$-adic unit. Thus

$$
t_{S_{1}}\left(c \dot{K}^{\ell}\right)=c x_{\mathfrak{q}} \dot{K}^{\ell} \equiv u_{\mathfrak{q}} \pi_{\mathfrak{q}} \dot{K}_{\mathfrak{q}}^{\ell}=t_{\mathfrak{p}}\left(u_{\mathfrak{p}} \dot{K}_{\mathfrak{p}}^{\ell}\right) \equiv t_{\mathfrak{p}}\left(c \dot{K}_{\mathfrak{p}}^{\ell}\right) \bmod \dot{K}_{\mathfrak{q}}^{\ell}
$$

If $z \in V$, then $z \in \dot{K}_{\mathfrak{p}}^{\ell}$ and from $\left(z, x_{\mathfrak{q}}\right)_{\mathfrak{q}}=\left(z, x_{\mathfrak{p}}\right)_{\mathfrak{p}}=1$ it follows $z \in \dot{K}_{\mathfrak{q}}^{\ell}$. Hence

$$
t_{S_{1}}\left(z \dot{K}^{\ell}\right)=z \dot{K}^{\ell} \equiv 1 \dot{K}_{\mathfrak{q}}^{\ell}=t_{\mathfrak{p}}\left(1 \dot{K}_{\mathfrak{p}}^{\ell}\right) \equiv t_{\mathfrak{p}}\left(z \dot{K}_{\mathfrak{p}}^{\ell}\right) \bmod \dot{K}_{\mathfrak{q}}^{\ell}
$$

Now suppose $\mathfrak{v} \in S_{1} \backslash\{\mathfrak{r}, \mathfrak{p}\}$. Obviously, for $z \in V$ the congruence in 4.6 holds. By the choice, the elements $x_{\mathfrak{p}}, x_{\mathfrak{q}}$ are local $\ell$ th powers in $\dot{K}_{\mathfrak{v}}$ and then

$$
\begin{aligned}
& t_{S_{1}}\left(x_{\mathfrak{p}} \dot{K}^{\ell}\right)=x_{\mathfrak{q}} \dot{K}^{\ell} \equiv 1 \dot{K}_{\mathfrak{v}}^{\ell}=t_{\mathfrak{v}}\left(1 \dot{K}_{\mathfrak{v}}^{\ell}\right) \equiv t_{\mathfrak{v}}\left(x_{\mathfrak{p}} \dot{K}_{\mathfrak{p}}^{\ell}\right) \bmod \dot{K}_{\mathfrak{v}}^{\ell}, \\
& t_{S_{1}}\left(c \dot{K}^{\ell}\right)=c x_{\mathfrak{q}} \dot{K}^{\ell} \equiv c \dot{K}_{\mathfrak{v}}^{\ell}=t_{\mathfrak{v}}\left(c \dot{K}_{\mathfrak{v}}^{\ell}\right) \bmod \dot{K}_{\mathfrak{v}}^{\ell} .
\end{aligned}
$$

We have just completed proving (4.6). Thus the triple $\left(T_{S_{1}}, t_{S_{1}}, \prod_{\mathfrak{v} \in S_{1}} t_{\mathfrak{v}}\right)$ is a small $S_{1}$-equivalence of degree $\ell$, for which $t_{\mathfrak{p}}$ is the only one wild isomorphism.

Analogously as in the case (I) one shows that this equivalence has an extension to a self-equivalence $(T, t)$ of degree $\ell$ of the field $K$ such that $\mathcal{W}(T, t)=\{\mathfrak{p}\}$.

\section{Summary}

In this section we present the proof of the main Theorem 1.1. We precede this proof with some remarks on the wild sets of a composition of two selfequivalences.

Suppose $K$ is a fixed number field containing a primitive $\ell$ th root of unity and $\left(T_{1}, t_{1}\right),\left(T_{2}, t_{2}\right)$ are fixed Hilbert self-equivalences of degree $\ell$ of $K$. To simplify the notation denote the wild sets $\mathcal{W}\left(T_{1}, t_{1}\right), \mathcal{W}\left(T_{2}, t_{2}\right)$ by $\mathcal{W}_{T_{1}}, \mathcal{W}_{T_{2}}$, respectively.

Of course, the pair $\left(T_{2} \circ T_{1}, t_{2} \circ t_{1}\right)$ is a Hilbert self-equivalence of degree $\ell$ of $K$. We shall describe the wild set $\mathcal{W}_{T_{2} \circ T_{1}}=\mathcal{W}\left(T_{2} \circ T_{1}, t_{2} \circ t_{1}\right)$ of the self-equivalence $\left(T_{2} \circ T_{1}, t_{2} \circ t_{1}\right)$. 
Suppose $\mathfrak{p}$ is a finite prime such that $\mathfrak{p} \notin \mathcal{W}_{T_{1}}$ and $T_{1} \mathfrak{p} \notin \mathcal{W}_{T_{2}}$. Then

$$
\operatorname{ord}_{T_{2} T_{1} \mathfrak{p}} t_{2} t_{1} x \equiv \operatorname{ord}_{T_{1} \mathfrak{p}} t_{1} x \equiv \operatorname{ord}_{\mathfrak{p}} x \quad(\bmod \ell) \quad \text { for every } x \in \dot{K} .
$$

Thus we clearly have the inclusion

$$
\mathcal{W}_{T_{2} \circ T_{1}} \subseteq T_{1}^{-1}\left(\mathcal{W}_{T_{2}}\right) \cup \mathcal{W}_{T_{1}}
$$

Now let $\mathfrak{p}$ be a finite prime such that $\mathfrak{p} \in \mathcal{W}_{T_{1}}, T_{1} \mathfrak{p} \notin \mathcal{W}_{T_{2}}$ and let $a \in \dot{K}$ be such that $\operatorname{ord}_{T_{1} \mathfrak{p}} t_{1} a \not \equiv \operatorname{ord}_{\mathfrak{p}} a(\bmod \ell)$. Then

$$
\operatorname{ord}_{T_{2} T_{1} \mathfrak{p}} t_{2} t_{1} a \equiv \operatorname{ord}_{T_{1} \mathfrak{p}} t_{1} a \not \equiv \operatorname{ord}_{\mathfrak{p}} a \quad(\bmod \ell),
$$

i.e. $\mathfrak{p}$ is a wild prime of the self-equivalence $\left(T_{2} \circ T_{1}, t_{2} \circ t_{1}\right)$.

Next assume $\mathfrak{p}$ is a finite prime such that $\mathfrak{p} \notin \mathcal{W}_{T_{1}}$ and $T_{1} \mathfrak{p} \in \mathcal{W}_{T_{2}}$, but $b=t_{1}(a) \in \dot{K}$ is such that $\operatorname{ord}_{T_{2} T_{1} \mathfrak{p}} t_{2} b \not \equiv \operatorname{ord}_{T_{1} \mathfrak{p}} b(\bmod \ell)$. Then

$$
\operatorname{ord}_{T_{2} T_{1} \mathfrak{p}} t_{2} t_{1} a \not \equiv \operatorname{ord}_{T_{1} \mathfrak{p}} t_{1} a \equiv \operatorname{ord}_{\mathfrak{p}} a \quad(\bmod \ell),
$$

i.e. $\mathfrak{p}$ is a wild prime of the self-equivalence $\left(T_{2} \circ T_{1}, t_{2} \circ t_{1}\right)$.

As a result we get the inclusion

$$
\left(\mathcal{W}_{T_{1}} \backslash T_{1}^{-1}\left(\mathcal{W}_{T_{2}}\right)\right) \cup\left(T_{1}^{-1}\left(\mathcal{W}_{T_{2}}\right) \backslash \mathcal{W}_{T_{1}}\right) \subseteq \mathcal{W}_{T_{2} \circ T_{1}},
$$

that is

$$
\left(\mathcal{W}_{T_{1}} \cup T_{1}^{-1}\left(\mathcal{W}_{T_{2}}\right)\right) \backslash\left(\mathcal{W}_{T_{1}} \cap T_{1}^{-1}\left(\mathcal{W}_{T_{2}}\right)\right) \subseteq \mathcal{W}_{T_{2} \circ T_{1}}
$$

COROLlaRY 5.1. Under the above assumptions and notations, if

$$
\mathcal{W}_{T_{1}} \cap T_{1}^{-1}\left(\mathcal{W}_{T_{2}}\right)=\emptyset
$$

then

$$
\mathcal{W}_{T_{2} \circ T_{1}}=\mathcal{W}_{T_{1}} \cup T_{1}^{-1}\left(\mathcal{W}_{T_{2}}\right)
$$

It is better to formulate this corollary in a more useful way.

Corollary 5.2. Assume $A, B$ are disjoint sets of finite primes of the field $K$. If $\left(T_{1}, t_{1}\right),\left(T_{2}, t_{2}\right)$ are Hilbert self-equivalences of degree $\ell$ of $K$ such that $\mathcal{W}\left(T_{1}, t_{1}\right)=A$ and $\mathcal{W}\left(T_{2}, t_{2}\right)=T_{1}(B)$, then $\mathcal{W}\left(T_{2} \circ T_{1}, t_{2} \circ t_{1}\right)=A \cup B$.

We use this corollary to prove the main theorem. 
Proof of Theorem 1.1. We use induction on $k$. For $k=1$ the statement follows from Theorem 4.1. Now suppose $k>1$. By Theorem 4.1, there exists a Hilbert self-eqivalence $\left(T_{1}, t_{1}\right)$ of degree $\ell$ of $K$ such that $\mathcal{W}\left(T_{1}, t_{1}\right)=$ $\left\{\mathfrak{p}_{1}\right\}$. Denote $\mathfrak{q}_{i}=T_{1} \mathfrak{p}_{i}$ for $i=2, \ldots, k$. By Proposition 3.11, the classes cl $\mathfrak{q}_{2}, \ldots, \mathrm{cl}_{k}$ are $\ell$ th powers in the group $C_{K}$. Thus the inductive assumption gives us a Hilbert self-equivalence $\left(T_{2}, t_{2}\right)$ of degree $\ell$ of $K$ such that $\mathcal{W}\left(T_{2}, t_{2}\right)=\left\{\mathfrak{q}_{2}, \ldots, \mathfrak{q}_{k}\right\}$. By Corollary 5.2, we obtain $\mathcal{W}\left(T_{2} \circ T_{1}, t_{2} \circ t_{1}\right)=$ $\left\{\mathfrak{p}_{1}\right\} \cup\left\{\mathfrak{p}_{2}, \ldots, \mathfrak{p}_{k}\right\}$.

\section{Final remarks}

Our main result required assumptions both on the field $K$ and the primes playing a role in the final statement. The fact that $K$ should contain a primitive $\ell$ th root of unity is unquestionable, but the necessity of the assumptions on the primes might be discussed. It is obvious that changing part of them will influence the final result. Below we give an example.

Let $K$ be a number field containing a primitive $\ell$ th root of unity (we do not assume $(\mathrm{C} 2)$ ). Notice that for any finite prime $\mathfrak{v}$ of $K$ the mapping $\varphi: \dot{K}_{\mathfrak{v}} / \dot{K}_{\mathfrak{v}}^{\ell} \rightarrow \dot{K}_{\mathfrak{v}} / \dot{K}_{\mathfrak{v}}^{\ell}, \varphi(x)=x^{\ell-1}$, is a group automorphism of $\dot{K}_{\mathfrak{v}} / \dot{K}_{\mathfrak{v}}^{\ell}$ which preserves Hilbert symbol of degree $\ell$. Indeed, for all $x, y \in \dot{K}_{\mathfrak{v}} / \dot{K}_{\mathfrak{v}}^{\ell}$ we have

$$
(\varphi(x), \varphi(y))_{\mathfrak{v}}=\left(x^{\ell-1}, y^{\ell-1}\right)_{\mathfrak{v}}=(x, y)_{\mathfrak{v}}^{(\ell-1)^{2}}=(x, y)_{\mathfrak{v}}
$$

Unfortunately, this automorphism is not tame.

Using the automorphisms defined above it is easy to define a Hilbert selfequivalence of degree $\ell$ for which the wild set is "relatively big". The following theorem shows such a case.

THEOREM 6.1. Let $K$ be a number field containing a primitive $\ell$ th root of unity. If the set $\left\{\mathfrak{p}_{1}, \ldots, \mathfrak{p}_{m}\right\}$ of finite primes of $K$ contains all $\ell$-adic primes and the ideal classes $\operatorname{cl} \mathfrak{p}_{1}, \ldots, c \mathrm{cl} \mathfrak{p}_{m}$ generate the group $C_{K} / C_{K}^{\ell}$, then there exists a Hilbert self-equivalence $(T, t)$ of degree $\ell$ of $K$ such that $\mathcal{W}(T, t)=$ $\left\{\mathfrak{p}_{1}, \ldots, \mathfrak{p}_{m}\right\}$.

Proof. Let $S_{\infty}$ be the set of all infinite primes of $K$ and let $S=S_{\infty} \cup$ $\left\{\mathfrak{p}_{1}, \ldots, \mathfrak{p}_{m}\right\}$. By Remark $2.2, \operatorname{rk}_{\ell} C_{S}=0$, i.e. the set $S$ is sufficiently large. 
Let us define the triple $\left(T_{S}, t_{S}, \prod_{\mathfrak{v} \in S} t_{\mathfrak{v}}\right)$ in the following way

$$
\begin{array}{ll}
T_{S}: S \rightarrow S, & T_{S}=\operatorname{id}_{S}, \\
t_{S}: E_{S} / \dot{K}^{\ell} \rightarrow E_{S} / \dot{K}^{\ell}, & t_{S}(x)=x^{\ell-1} \\
t_{\mathfrak{v}}: \dot{K}_{\mathfrak{v}} / \dot{K}_{\mathfrak{v}}^{\ell} \rightarrow \dot{K}_{\mathfrak{v}} / \dot{K}_{\mathfrak{v}}^{\ell}, & t_{\mathfrak{v}}(x)=x^{\ell-1} \quad \text { for every } \mathfrak{v} \in S .
\end{array}
$$

Obviously, $\left(T_{S}, t_{S}, \prod_{\mathfrak{v} \in S} t_{\mathfrak{v}}\right)$ is a small $S$-equivalence of degree $\ell$ of $K$, which, by Theorem 3.12, can be extended to a self-equivalence $(T, t)$ of degree $\ell$ of $K$ and tame outside $S$. Every finite prime $\mathfrak{v}$ in $S$ is wild, so $\mathcal{W}(T, t)=$ $\left\{\mathfrak{p}_{1}, \ldots, \mathfrak{p}_{m}\right\}$.

The notion of a tame prime of a Hilbert semi-equivalence of degree $\ell(\ell-$ prime number) came into existence as a natural generalization of the same notion for a Hilbert self-equivalence of degree 2. In the case of $\ell>2$ it seems interesting to consider also another generalization which we shall describe below.

Suppose $(T, t)$ is a Hilbert self-equivalence of degree $\ell$ of $K$. A finite prime $\mathfrak{p}$ of $K$ is called semi-tame if

$$
\forall_{a \in \dot{K} / \dot{K}^{\ell}}\left(\operatorname{ord}_{\mathfrak{p}} a \equiv 0 \quad(\bmod \ell) \Longleftrightarrow \operatorname{ord}_{T \mathfrak{p}} t a \equiv 0 \quad(\bmod \ell)\right) .
$$

A finite prime $\mathfrak{p}$ is called semi-wild if it is not semi-tame. The set of all semi-tame primes of a Hilbert self-equivalence $(T, t)$ is called semi-wild and denoted by $\mathcal{S W}(T, t)$.

Of course, every tame prime is semi-tame, but the opposite implication is not true. Thus the following inclusion:

$$
\mathcal{S} \mathcal{W}(T, t) \subseteq \mathcal{W}(T, t)
$$

In the same way as in the case of a tame isomorphism one can define the notion of a semi-tame local isomorphism. For finite primes $\mathfrak{p}$ and $\mathfrak{p}^{\prime}$ of $K$ a local isomorphism $\varphi: \dot{K}_{\mathfrak{p}} / \dot{K}_{\mathfrak{p}}^{\ell} \rightarrow \dot{K}_{\mathfrak{p}^{\prime}} / \dot{K}_{\mathfrak{p}^{\prime}}^{\ell}$ preserving Hilbert symbols of degree $\ell$ is said to be semi-tame, if

$$
\forall_{x \in \dot{K}_{\mathfrak{p}} / \dot{K}_{\mathfrak{p}}^{\ell}}\left(\operatorname{ord}_{\mathfrak{p}} x \equiv 0 \quad(\bmod \ell) \quad \Longleftrightarrow \quad \operatorname{ord}_{\mathfrak{p}^{\prime}} \varphi(x) \equiv 0 \quad(\bmod \ell)\right) .
$$

and semi-wild, otherwise.

It is a routine matter to check that if $(T, t)$ is a Hilbert self-equivalence of degree $\ell$ of $K$, then a finite prime $\mathfrak{p}$ is semi-tame if and only if the local isomorphism $t_{\mathfrak{p}}: \dot{K}_{\mathfrak{p}} / \dot{K}_{\mathfrak{p}}^{\ell} \rightarrow \dot{K}_{T \mathfrak{p}} / \dot{K}_{T \mathfrak{p}}^{\ell}$ induced by $t$ is semi-tame. 
Analyzing the proof of Theorem 1.1 we notice that the local isomorphisms $t_{\mathfrak{p}}$ constructed above are semi-tame. This leads us to the following theorem, which is a stronger version of Theorem 1.1 .

THEOREM 6.2. Let $\ell>2$ be a prime number and let $K$ be a number field satisfying $(\mathrm{C} 1)$ and $(\mathrm{C} 2)$. If $W=\left\{\mathfrak{p}_{1}, \ldots, \mathfrak{p}_{k}\right\}$ is a set of finite primes of $K$ and the classes $\mathrm{cl} \mathfrak{p}_{1}, \ldots \mathrm{cl} \mathfrak{p}_{k}$ are $\ell$ th powers in $C_{K}$, then there exists a Hilbert self-equivalence $(T, t)$ of degree $\ell$ of $K$ such that $\mathcal{S W}(T, t)=W$.

In the proof of Theorem 6.1 all local isomorphisms $t_{\mathfrak{v}}$ for $\mathfrak{v} \in S$ constructed there are semi-tame, so similar strengthening of Theorem 6.1 is not possible.

The problem of describing all finite sets of primes which are wild sets or semi-wild sets of Hilbert self-equivalences of degree $\ell>2$ is open.

\section{References}

[1] Cassels J.W., Fröhlich A., Algebraic Number Theory, Academic Press, London, 1967.

[2] Czogała A., Sładek A., Higher degree Hilbert symbol equivalence of number fields, Tatra Mountains Math. Publ. 11 (1997), 77-88.

[3] Czogała A., Sładek A., Higher degree Hilbert symbol equivalence of number fields II, J. Number Theory 72 (1998), 363-376.

[4] Czogała A., Higher degree tame Hilbert-symbol equivalence of number fields,. Abh. Math. Sem. Univ. Hamburg 69 (1999), 175-185.

[5] Czogała A., On reciprocity equivalence of quadratic number fields, Acta Arith. 58 (1991), 27-46.

[6] Czogała A., Witt rings of Hasse domains of global fields, J. Algebra 244 (2001), 604630.

[7] Czogała A., Rothkegel B., Wild primes of a self-equivalence of a number field, Acta Arith. 166 (2014), 27-46.

[8] Leep D.B., Wadsworth A.R., The Hasse norm theorem mod squares, J. Number Theory 42 (1991), 337-348.

[9] Milnor J., Algebraic K-Theory and quadratic forms, Invent. Math. 9 (1970), 318-344.

[10] Neukirch J., Class Field Theory, Springer-Verlag, Berlin, 1986.

[11] Perlis R., Szymiczek K., Conner P., Litherland R., Matching Witts with global fields, Contemp. Math. 155 (1994), 365-387.

[12] Rothkegel B., Czogała A., Singular elements and the Witt equivalence of rings of algebraic integers, Ramanujan J. 17 (2008), 185-217.

[13] Somodi M., On the size of the wild set, Canad. J. Math. 55 (2005), 180-203.

[14] Somodi M., A characterization of the finite wild sets of rational self-equivalences, Acta Arith. 121 (2006), 327-334.

[15] Somodi M., Self-equivalences of the Gaussian field, Rocky Mountain J. Math. 38 (2008), 2077-2089.

[16] Sładek A., Hilbert symbol equivalence and Milnor K-functor, Acta Math. Inform. Univ. Ostraviensis 6 (1998), 183-190. 
Institute of Mathematics

University of Silesia

BANKOWA 14

40-007 Katowice

POLAND

e-mail: alfred.czogala@us.edu.pl

e-mail: beata.rothkegel@us.edu.pl

e-mail: andrzej.sladek@us.edu.pl 